\title{
GENERATION OF PRESSURE BY EWOD-ACTUATED DROPLETS
}

\author{
J. Jenkins ${ }^{*}$ and C.-J. Kim
}

\author{
Mechanical and Aerospace Engineering Department, University of California, Los Angeles (UCLA) \\ Los Angeles, California, U.S.A.
}

\begin{abstract}
We demonstrate the accumulation of pumping pressure developed by multiple droplets in series actuated simultaneously by electrowetting-on-dielectric (EWOD). To measure pressure generated by EWOD, water droplets in a silicone oil medium are confined to a microchannel and driven together against a known pneumatic pressure until they can no longer advance forward. The total pressure withstood is found to scale approximately linearly with the number of droplets. Using $80 \mathrm{~V}_{\mathrm{AC}}$ at $1 \mathrm{kHz}$, while a single droplet was measured to develop $1.4 \mathrm{kPa}$ of pressure, up to 6 droplets together generated $7.6 \mathrm{kPa}$, opening the door for EWOD to be used as a pumping mechanism in high pressure applications.
\end{abstract}

\section{INTRODUCTION}

Electrowetting-on-dielectric (EWOD) [1-2] is a proven scheme for microfluidic actuation, especially popular for dropletbased digital microfluidics [3-7] in lab-on-a-chip [5-10] as well as other [11-14] applications. It carries the advantages of low power consumption and small liquid volume requirements, as it directly manipulates discrete individual droplets. The ability to move droplets around a device area atop patterned electrodes has evolved the essential droplet functions of dispensing, translation, splitting and mixing [3-5]. Most applications derive their functionality by combining these functions to perform higher order tasks. At the core of EWOD actuation, however, is a pressure gradient, internal to the droplet, which gives rise to its motion. In our experiments, we harness this pressure to work against an external pressure, demonstrating EWOD's value not only as a means of displacing a droplet of interest from one place to another, but also as a viable mechanism for pumping other media at elevated pressures.

In a typical EWOD device configuration (Figure 1), voltage is applied between electrodes above and beneath a liquid droplet, at least one of which is covered by a thin dielectric layer. The presence of the electric field tightens (increases) the curvature of the liquid meniscus at the liquid-solid contact line, where the electric field is highly concentrated, and the apparent contact angle of the liquid on the solid surface decreases [15, 16], which is equivalent to the surface becoming more wetting to the liquid. Actuating an electrode that sits beneath only a portion of the contact line results in a disparity in meniscus curvature from one end of the droplet to the other, and hence, an internal pressure gradient. Calculations have predicted this pressure to be as high as $9 \mathrm{kPa}$ at $100 \mathrm{~V}$ for a typical device configuration under ideal conditions [1] (that is, without taking contact angle hysteresis into account), but have not been rigorously confirmed by experimental measurements on devices of this kind. Furthermore, early work on continuous electrowetting suggested that the pressure drops across individual droplets placed in series could theoretically be accumulated [17], transmitting the effect of each through a surrounding medium to its neighbor, but this effect had yet to be proven or explored in experiments. In order to use EWOD actuation for pumping with wide applicability, including devices with large resistance, back pressure, or perhaps to pump ancillary media by driving against a deflecting membrane [18], we need to first verify that the total pumping pressure available indeed increases with the number of droplets actuated and then test whether the correlation between pressure and number of droplets is one of strict summation.

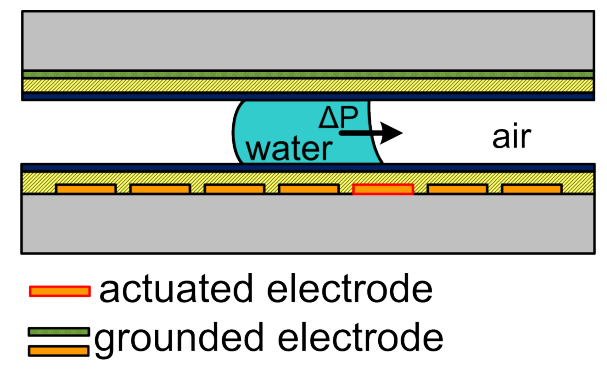

Figure 1: Cross-section schematic of typical EWOD device configuration.

Although EWOD is usually used to move a liquid volume of interest itself, some have shown that it is suitable for use more generally as pump components analogous to pistons and valves [19]. Microchannels filled with dodecane oil contained water droplets strategically positioned and held in place by constrictions in channel width and height. Under actuation of $65 \mathrm{~V}_{\mathrm{DC}}$ to $80 \mathrm{~V}_{\mathrm{DC}}$, the droplet menisci were able to advance into the narrower segments. This mechanism was employed with an appropriate actuation sequence such that some droplets acted as inlet and outlet valves, opening or blocking the channel depending on actuation state. Another larger droplet acted as a piston, advancing into the flow channel to displace oil through the open valves. In this way, significant flow rates of the oil medium were achieved.

Yet other work showed the development of a micropump based on oscillating continuous electrowetting actuation of a mercury droplet within a microchannel [18]. The device consisted of components fabricated from three substrates stacked together, forming a microchannel for the mercury droplet, silicone rubber membranes deflected by the droplet motion, and inlet and outlet chambers with metallic check valves, into which water was drawn and later expelled by the deflecting membrane.

Since driving pressure increases with actuation voltage, higher pressures could be achieved using one droplet alone and increasing the voltage applied, however, such an approach is significantly limited. High actuation voltage leads to the wellknown contact angle saturation, electrolysis and eventually dielectric breakdown. Adding droplets rather than increasing the actuation voltage avoids these problems while maintaining relatively low power consumption. Our work presents a micropumping scheme that offers a simple device with versatility, convenience, and a capability of vastly higher output pressure.

\section{EXPERIMENTAL PROCEDURE \\ Device Fabrication}

To direct accumulated pressure, a microchannel is fabricated atop an otherwise common-configuration EWOD device. As shown in Figure 2(a), $\mathrm{Cr} / \mathrm{Au}$ electrodes and contact pads are patterned on a glass substrate, laid out in a long row. This is followed by deposition of a $1.2 \mu \mathrm{m}$ PECVD $\mathrm{Si}_{\mathrm{x}} \mathrm{N}_{\mathrm{y}}$ dielectric layer. A $50 \mu \mathrm{m}$ thick $\mathrm{KMPR}^{\circledR} 1050$ (MicroChem) layer is then spincoated and patterned to form channel walls. A glass slide coated with indium tin oxide (ITO) to act as the grounding electrode serves as a cover plate, shown in Figure 2(b). A $100 \mathrm{~nm}$ thick 


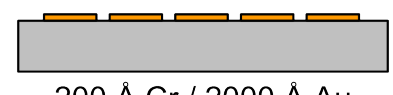

$200 \AA \mathrm{A} \mathrm{Cr} / 2000 \AA \mathrm{Au}$ deposited and patterned

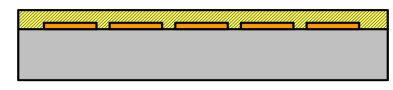

$1.2 \mu \mathrm{m}$ silicon nitride deposited by PECVD

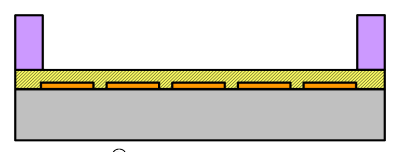

KMPR $^{\circledR} 1050$ spin-coated and patterned

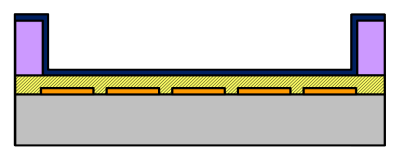

Cytop hydrophobic coating spin-coated

(a)

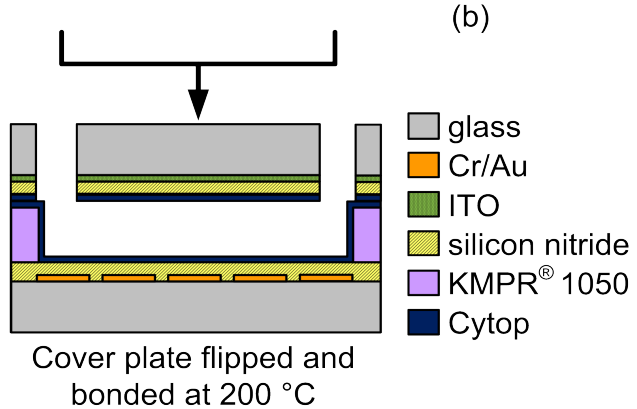

(c)

Figure 2: Process flow for (a) device substrate, (b) cover plate, and (c) substrate-cover assembly. Note the sidewall, not generating EWOD force, only retards the motion.

$\mathrm{Si}_{\mathrm{x}} \mathrm{N}_{\mathrm{y}}$ layer is deposited on the cover plate for isolation. Next, access holes $1 \mathrm{~mm}$ in diameter are drilled in the cover plate to allow the later addition of water droplets and oil medium. A hydrophobic coating of $2 \%$ Cytop solution is spin-coated on both the device substrate and cover plate. The cover plate is flipped, aligned to the substrate as in Figure 2(c), and clamping force is applied to the assembly on a hot plate at $200{ }^{\circ} \mathrm{C}$, allowing the Cytop to reflow and bond the cover plate to the substrate. Finally, tubing is epoxied over the drilled access holes for liquid filling.

\section{Pressure Measurement}

The pressure generated is determined by equilibrating actuated droplets against a known external source. We use a tank of compressed nitrogen with a finely controlled regulator to adjust this backpressure supplied to the outlet of the device microchannel. The channel is first filled with silicone oil to act as an effective medium for pressure transmission between the droplets. Before applying any pressure from the nitrogen tank, deionized water droplets are dispensed from a reservoir by EWOD and moved into position in the microchannel individually, arranged in series and with at least one grounded electrode space between them to avoid unintentional actuation in the reverse direction.
With the inlet behind the train of droplets left open to atmosphere throughout the experiments, the pressure from the tank is steadily increased through the regulator. When the supplied backpressure is sufficient to overcome the droplet's friction force (i.e., static resistance) at the channel walls, they slowly begin to move backward. The droplets are then driven against the tank pressure by EWOD with $80 \mathrm{~V}_{\mathrm{AC}}$ at $1 \mathrm{kHz}$. In our device, the sidewalls of the channel are passive, and do not contribute to the droplet's pressure development. On the contrary, due to friction, the presence of the sidewalls retards the motion as compared to an open EWOD configuration, requiring higher voltage to overcome. Recent work has shown that electrodes may be incorporated into the channel sidewalls [20], aiding in the forward driving force, and thus reducing the applied voltage necessary to move droplets. However, such a configuration requires a significantly more complex fabrication process including the production of a shadow mask with microscale resolution by front- and backside throughwafer etching.

We confirmed experimentally that water, with a contact angle on Cytop of approximately $110^{\circ}$, completely fills the channel corners when in an air medium (Figure 3(a)). A droplet was placed in a passive microchannel and pressure was applied to one end by way of the nitrogen tank. A digital pressure gauge was connected to the outlet of the channel and the pressure on this side of the droplet was monitored over time. With $3 \mathrm{kPa}$ of applied pressure to the inlet, no pressure change aft of the droplet was detected for several hours, implying that the droplet completely filled the corners of the channel, letting none of the applied pressure leak past it.

(a)
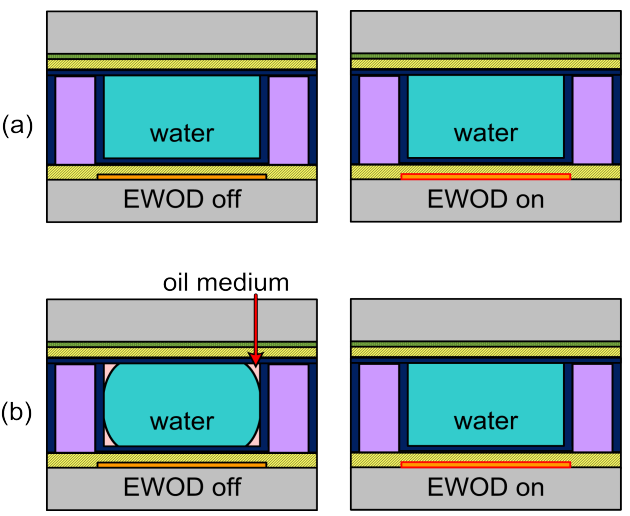

Figure 3: Cross-section schematic (showing a "front view" of the droplet, orthogonal to the cross-section plane of Figure 2) of water droplet (a) in air medium over unactuated (left) and actuated (right) electrodes, (b) in oil medium over unactuated (left) and actuated (right) electrodes. In air medium (a), water (contact angle $\sim 110^{\circ}$ ) fills the corners naturally with or without EWOD actuation, which was confirmed by experiments. In oil medium (b), on the other hand, water (contact angle $\sim 160^{\circ}$ ) does not fill the corners unless EWOD is on.

However, during our cumulative pressure generation experiments, we found that actuating series of droplets primarily compressed the volume of air trapped between them in the channel, rather than contributing additional total pumping pressure. For this reason, we performed our experiments in a medium of silicone oil, whose incompressibility allowed for effective transmission of pressure from one droplet to the next. In trade-off, however, the water makes a much higher contact angle with the channel walls, $\sim 160^{\circ}$, which fails to fill the corners (Figure 3(b)) 
when no actuation voltage is applied. To overcome the potential problem of pressure leak through the corners, we have modified the electrode actuation scheme as compared to typical EWOD devices: at least three electrodes are allotted per droplet as shown in Figure 4. The electrode under each droplet's leading meniscus is actuated as well as the one beneath its midsection. The former induces the droplet to wet and advance along the leading electrode. The latter does not provide any driving pressure, but reduces the effective contact angle at the channel surfaces to help spread the droplet to the full channel width, aiding in preventing leakage of the surrounding medium past the droplet (Figure 3(b)). The electrode under the droplet's trailing meniscus is grounded so as to remain in a hydrophobic state, establishing the droplet driving pressure.

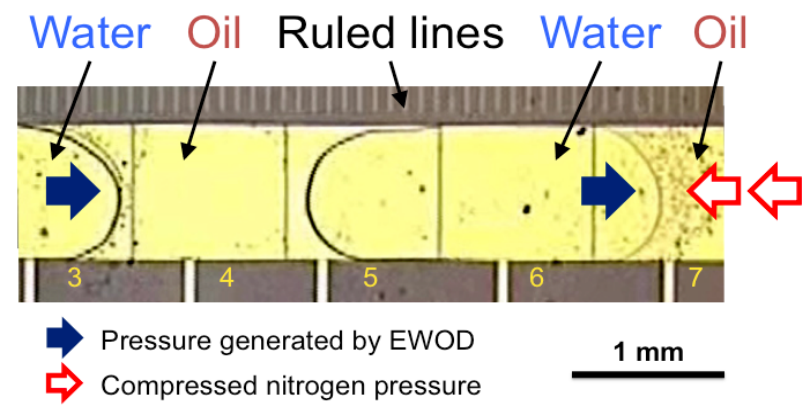

Figure 4: Top view photo of two droplets actuated simultaneously by EWOD to oppose a pressure from right. Electrodes 2 (not shown), 3, 6 and 7 are actuated with $80 V_{A C}$ at $1 \mathrm{kHz}$, while electrodes 1 (not shown), 4 and 5, as well as the transparent top cover plate are grounded. Ruled lines help to identify point of droplet stalling.

Actuation of multiple droplets together is observed to counteract higher tank pressures, which is adjusted until the droplets stall (Figure 4). Ruled lines patterned on the surface of the device help to identify the moment at which the droplets are no longer able to move forward against the compressed nitrogen, providing a visual stationary reference point. At the stalling pressure, removing voltage from any one of the droplets allows the entire group to move backward down the channel under the backpressure. Reinstating voltage prevents the loss of further ground. The balanced pressure is read from a digital gauge connected to a parallel on-chip channel, crosschecked with the reading of the inlet regulator, and recorded. This pressure is reported as that generated by the procession of droplets. A side view schematic of the experimental setup is shown in Figure 5.

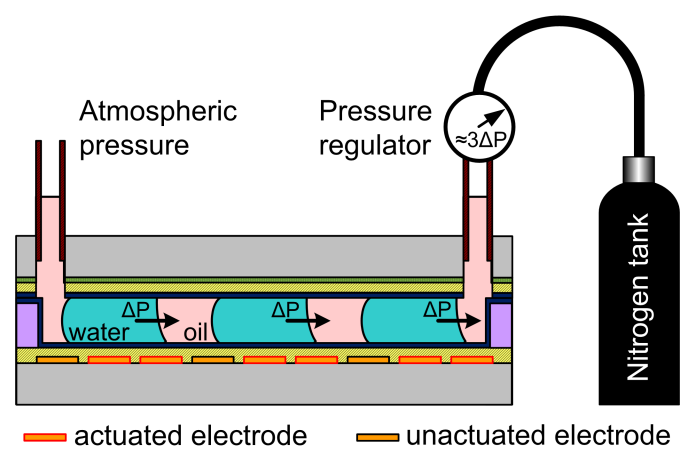

Figure 5: Side view schematic of experimental setup showing multiple droplets (3 shown) in series pushing against the regulated pressure.

\section{RESULTS AND DISCUSSION}

Measured pressure results are plotted versus the number of droplets actuated in series in Figure 6, along with a linear curve fit. Each point represents an average of four experimental measurements for each series of droplets. The data show that with each additional droplet, the total pressure withstood increases nearly proportionally. In these experiments, each droplet contributes approximately $1.4 \mathrm{kPa}$ of pressure to the total. The frictional hindrance to droplet motion due to the channel sidewalls appears to be constant for each droplet; the pressure increase by each additional actuated droplet does not appear to diminish at higher numbers of droplets. With up to six droplets, the output pressure of $7.6 \mathrm{kPa}$ achieved is higher than the $2.0-2.5 \mathrm{kPa}$ seen by the aforementioned single piston EWOD-based pumping system under similar voltage [19], on account of our multiple droplets working together. Extrapolation of the plot implies that even higher pressures for more demanding applications can be achieved simply through the actuation of a greater number of droplets.

\section{Total pressure generated vs. number of droplets}

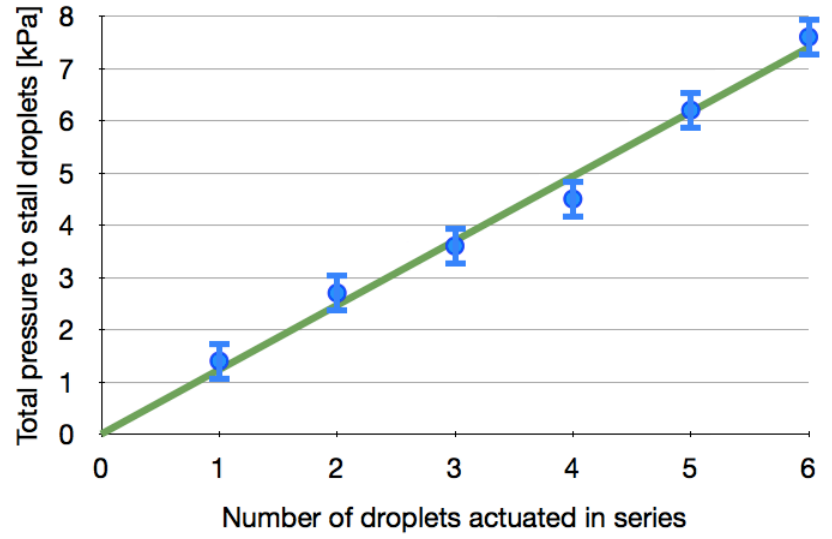

Figure 6: Plot of mean total accumulated pressure versus number of droplets in series actuated by EWOD simultaneously. Error bars represent variation in gauge reading across 4 experiments for each point.

Expected performance was calculated from the LippmannYoung and Young-Laplace equations that are often used to describe EWOD actuation [1,2]. For the electrode configuration used in our experiments, the resulting equation for internal droplet pressure gradient $\Delta P$ is given as:

$$
\Delta P=\frac{1}{2} \frac{\varepsilon \varepsilon_{o} V^{2}}{t d}
$$

where $\varepsilon$ is the dielectric constant of the dielectric layer, $\varepsilon_{\mathrm{o}}$ is the permittivity of free space, $V$ is applied voltage, $t$ is the thickness of the dielectric layer and $d$ is the microchannel height.

Frictional resistance forces resulting from contact with the substrate, cover plate and channel sidewalls were taken into account, approximated for each surface using contact angle hysteresis force [21], $F_{\text {hyst }}$, which can be written as:

$$
F_{\text {hyst }}=w \sigma\left(\cos \theta_{r}-\cos \theta_{a}\right)
$$

where $w$ is the width of the contact area between the liquid and solid surface, $\sigma$ is the interfacial tension between the liquid and the surrounding medium, and $\theta_{r}$ and $\theta_{a}$ are receding and advancing 
contact angles, respectively.

With $50 \mu \mathrm{m}$ channel height, $1.4 \mathrm{~mm}$ channel width, $80 \mathrm{~V}_{\mathrm{AC}}$ actuation voltage, $1.2 \mu \mathrm{m}$ thick dielectric layer, material properties for silicon nitride and the approximate receding and advancing contact angles of $158^{\circ}$ and $162^{\circ}$, respectively, for Equations 1 and 2 , each droplet is calculated to withstand a pressure of $3.9 \mathrm{kPa}$. The measured value of approximately $1.4 \mathrm{kPa}$ per droplet represents $36 \%$ of this expected value. We anticipate that improvements in the quality of fabrication, especially in the smoothness of the surfaces will bring experimental results closer in line with their theoretical values. Spin-coating of the Cytop hydrophobic layer on features as tall as the channel sidewalls may result in non-uniform coverage. Furthermore, Cytop reflow during the bonding of the cover plate may have caused pooling in the corners of the channel.

\section{CONCLUSIONS}

We have shown that high pressure pumping can be achieved using EWOD actuation of droplets. While each droplet exerts a pressure as a function of the EWOD voltage applied, the total output pressure of such a micropumping device is a function of the number of droplets simultaneously actuated. We observed that pressure sums from one droplet to the next in series, measuring a total pressure for up to six droplets that was approximately six times that measured for a single droplet. As such, voltage need not be increased to develop high pumping pressures, but may be fixed to that which is allowed or available by the device in hand or required by the application. Devices may be designed with a large number of droplets to meet pressure needs, with the ability to increase or decrease pressure as necessary by simply controlling the signal to the electrodes, turning the contributions of certain individual droplets on or off. Seemingly limited only by fabrication capabilities, current values suggest even a full atmosphere of pumping pressure could be realized with 70 droplets pumping together.

\section{ACKNOWLEDGEMENTS}

The authors would like to thank Dr. Wyatt Nelson, Dr. Prosenjit Sen and all the members of the UCLA Micro and Nano Manufacturing Laboratory for their helpful advice and discussion.

\section{REFERENCES}

[1] J. Lee, H. Moon, J. Fowler, T. Schoellhammer and C.-J. Kim, "Electrowetting and electrowetting-on-dielectric for microscale liquid handling", Sensor. Actuat. A-Phys., 95, 259 (2002).

[2] H. Moon, S.K. Cho, R.L. Garrell, and C.-J. Kim, "Low voltage electrowetting-on-dielectric", J. Appl. Phys., 92, 4080 (2002).

[3] S.K. Cho, H. Moon, and C.-J. Kim, "Creating, Transporting, Cutting, and Merging Liquid Droplets by ElectrowettingBased Actuation for Digital Microfluidic Circuits", J. Microelectromech. Syst., 12, 70 (2003).

[4] M.G. Pollack, A.D. Shenderov, and R.B. Fair, "Electrowetting-Based Actuation of Droplets for Integrated Microfluidics", Lab Chip, 2, 96 (2002).

[5] P. Paik, V.K. Pamula, and R.B. Fair, "Rapid droplet mixers for digital microfluidic systems", Lab Chip, 3, 253 (2003).

[6] H. Moon, A.R. Wheeler, R.L. Garrell, J.A. Loo, and C.-J. Kim, "An integrated digital microfluidic chip for multiplexed proteomic sample preparation and analysis by MALDI-MS", Lab Chip, 6, 1213 (2006).
[7] V. Srinivasan, V.K. Pamula, and R.B. Fair, "An integrated digital microfluidic lab-on-a-chip for clinical diagnostics on human physiological fluids", Lab Chip, 4, 310 (2004).

[8] P.Y. Keng, S. Chen, H. Ding, S. Sadeghi, G.J. Shah, A. Dooraghi, M.E. Phelps, N. Satyamurthy, A.F. Chatziioannou, C.-J. Kim, and R.M. van Dam, "Micro-chemical synthesis of molecular probes on an electronic microfluidic device", Proc. Natl. Acad. Sci. USA, 109, 690 (2012).

[9] W.C. Nelson, I. Peng, G.-A. Lee, J.A. Loo, R.L. Garrell, and C.-J. Kim, "Incubated Protein Reduction and Digestion on an Electrowetting-on-Dielectric Digital Microfluidic Chip for MALDI-MS", Anal. Chem., 82, 9932 (2010).

[10] N. Vergauwe, D. Witters, F. Ceyssens, S. Vermeir, B. Verbruggen, R. Puers, and J. Lammertyn, "A Versatile Electrowetting-Based Digital Microfluidic Platform for Quantitative Homogeneous and Heterogeneous Bio-Assays", J. Micromech. Microeng., 21, 054026 (2011).

[11] P. Sen and C.-J. Kim, "A Fast Liquid-Metal Droplet Microswitch Using EWOD-Driven Contact-Line Sliding”, J. Microelectromech. Syst., 18, 174 (2009).

[12] W.C. Nelson, H.P. Kavehpour, and C.-J. Kim, “A miniature capillary breakup extensional rheometer by electrostatically assisted generation of liquid filaments", Lab Chip, 11, 2424 (2011).

[13] Y.-Y. Lin, C.-W. Lin, L.-J. Yang, and A.-B. Wang, "Microviscometer based on electrowetting on dielectric", Electrochim. Acta, 52, 2876 (2007).

[14] P. Mach, T. Krupenkin, S. Yang, and J. Rogers, "Dynamic tuning of optical waveguides with electrowetting pumps and recirculating fluid channels", Appl. Phys. Lett., 81, 202 (2002).

[15] F. Mugele and J. Buehrle, "Equilibrium drop surface profiles in electric fields", J. Phys.: Condens. Matter, 19, 375112 (2007).

[16] W.C. Nelson and C.-J. Kim, "Droplet Actuation by Electrowetting-on-Dielectric: A Review", J. Adhes. Sci. Technol., In press.

[17] E. Colgate, H. Matsumoto, "An investigation of electrowetting-based actuation", J. Vac. Sci. Technol. A, 8, 3625 (1990).

[18] K.-S. Yun, I.-J. Cho, J.-U. Bu, C.-J. Kim, and E. Yoon, "A surface-tension driven micropump for low-voltage and lowpower operations", J. Microelectromech. Syst., 11, 454 (2002).

[19] J. Kedzierski, S. Berry, and B. Abedian, "New Generation of Digital Microfluidic Devices", J. Microelectromech. Syst., 18, 845 (2009).

[20] S. Morishita, M. Kubota, and Y. Mita, "Integration of EWOD pumping device in deep microfluidic channels using a threedimensional shadowmask", Proc Int. Conf. MEMS, Paris, France, 1/29-2/2, IEEE (2012), pp. 1045-1048.

[21] E.B. Dussan V. and R.T.-P. Chow, "On the ability of drops or bubbles to stick to non-horizontal surfaces of solids", J. Fluid Mech., 137, 1 (1983).

\section{CONTACT}

*J. Jenkins, tel: +1-310-825-3977; jamesrj@UCLA.edu 